I Universidad de Chile, Facultad de Ciencias Sociales, Departamentos de Antropología y de Trabajo Social, Santiago de Chile, Chile. hcadenas@uchile.cl https://orcid.org/oooo-0003-2499-2430

I I Centro de Estudios Públicos, Santiago de Chile, Chile Hugo Cadenas' amascareno@cepchile.cl https://orcid.org/0000-0002-5803-863X Aldo Mascareño"

\title{
LINEAMIENTOS PARA UNA SOCIOLOGÍA EVOLUTIVA DE LA DIFERENCIACIÓN FUNCIONAL EN AMÉRICA LATINA
}

\section{INTRODUCCIÓN ${ }^{1}$}

El problema de la diferenciación funcional en América Latina ha sido visitado con regularidad desde las teorías de la modernización (Germani, I962; I98I). En este marco conceptual, que en última instancia responde a una estilización de las categorías weberianas y parsonianas relativas al surgimiento de la sociedad moderna, la región de América Latina era vista, fundamentalmente, mediante una distinción que destacaba la completitud de los países o regiones desarrolladas y la incompletitud latinoamericana. Se miraba una transición evolutiva, pero se la pensaba fundamentalmente en términos unilineales. No es que no hubiese espacio para estados intermedios o líneas alternativas de desarrollo. Estos se presentaban en múltiples variedades: desde formaciones híbridas, como la democracia con participación limitada, hasta variantes evolutivas que coexisten en una misma temporalidad, como todo el análisis que hacía referencia a la asincronía institucional, es decir, a expectativas y comportamientos que, en el marco de la teoría, correspondían a épocas distintas, como por ejemplo el afán de lucro frente al comportamiento ritual.

El marxismo tampoco dejó de lado la diferenciación funcional en América Latina, aun cuando la llamó clásicamente 'división del trabajo', con lo cual logró pensarla a través de una conceptualización histórica, pero no en términos evolutivos (e.g. Mariategui, 2007). Para dicho enfoque, el problema era cómo incluir a los "indígenas" en la división del trabajo si estos no eran ni proletarios 
ni burgueses, ya que la teoría europea obligaba a su inserción en alguno de los lados de la distinción. De todos modos, se los sabía explotados y sin conciencia de clase. Se sabía también que migraban del campo a la ciudad y que ahí se convertían en marginalizados o, si tenían suerte y lograban educarse, en alienados miembros de una sociedad burguesa que nunca los consideraría propios (Jaguaribe, 1976). Todo ello importaba para considerar las condiciones que América Latina ofrecía para la revolución. Parecía que el pueblo estaba enfrentado consigo mismo y había que unirlo; las razas estaban desencontradas (Ribeiro, 2008); la situación de explotación era demasiado moderna para quienes la vivenciaban por medio del ritual en vez de por la división del trabajo. En este sentido, el marxismo fue más bien inofensivo. La única experiencia de unión exitosa fue la chilena entre los años I 970 y I973, pero esta tuvo lugar bajo condiciones de ascenso de clases bajas, producto de una legislación social ampliada con condiciones estructurales y políticas relativamente estables, las mismas que hicieron fracasar el proyecto con el golpe militar de 1973.

De manera subsidiaria a la teoría de la modernización y al marxismo, la teoría de la dependencia representó una variante progresista. Tampoco podía llegar a extremos, porque su marco institucional eran las Naciones Unidas - a través de Cepal - y ya se veían los límites del marxismo en América Latina con el populismo brasileño y argentino. El análisis de Fernando Henrique Cardoso y Enzo Faleto (I990) reconocía el aporte de las teorías de la modernización, pero las consideraba incompletas por la falta de "componente histórico", con el que se entendía un concepto marxista de historia, una especie de filosofía de la historia que enfatizaba el rol de actores sociales y relaciones clásicas de poder como causalidad entre ellos: alguien detenta el poder y lo emplea frente a otro que no lo tiene, o lo tiene en menor medida, para conseguir sus propios intereses. Con esta teoría del poder de fondo, la teoría de la dependencia pretendía explicar las desigualdades y la dependencia entre centros europeos y americanos y la periferia latinoamericana. Es decir, América Latina se encontraba en una posición subordinada a los centros mundiales, básicamente, "porque tenía menos poder". Con ello se producía una explicación de tipo circular: la periferia era dependiente de los centros porque los centros la dominaban. De ello sacaban consecuencias que no dejaban de ser sugestivas y empíricamente fundadas, como la reproducción de la distinción centro/periferia al interior de las periferias, o como los límites de la autonomía en términos de conocimiento y tecnología que hacían fracasar la industrialización de la periferia. Sin embargo, la teoría estaba impedida de un acercamiento evolutivo o histórico que no significara historia en el sentido marxista del término: actores en disputa anclados en relaciones de producción, orientación a una finalidad, asimetrías de poder y subvaloración moral de todo lo que no se leyera bajo estas categorías.

El cuarto momento de las teorías latinoamericanas, uno que ha perdurado hasta la actualidad en distintas formas, es el de las teorías culturalistas 
de la identidad. De estas hay para todos los gustos y bolsillos. Aquí la diferenciación funcional y la evolución de la sociedad moderna se pierden en la alienación, mientras la identidad adquiere un núcleo categorial fundamental e inamovible desde la conquista (Morandé, I987), o queda oculta como estructura sobre la que se sustenta un desarrollo económico y modernidad epitelial, donde la identidad - de todos modos móvil - mueve la agencia en un sentido u otro (Larraín, 2004), o permanece como imposición colonial que produce una herida epistemológica, obligando a "volver a las raíces" para poder ser cerrada (Mignolo, 2005).

Cada una de estas versiones tiene derivados. Las teorías de la modernización como alienación creen que se puede pensar a América Latina como funcionalmente diferenciada, pero lo hacen en los términos europeos, sin apropiación histórica del proceso y sin realmente pensarlo evolutivamente. Por otro lado, creen que se puede hablar de la diferenciación de sistemas en América Latina y al mismo tiempo mantener a la persona y la familia como núcleo irreductible de la sociedad, en el modo del catolicismo más ingenuo (Valenzuela \& Cousiño, 20I5). Las teorías del desarrollo, en tanto, han pasado de los obstáculos estructurales al desarrollo económico (Fajnzylber, I983; Véliz, I970), al desarrollo humano, y a diagnosticar - nuevamente con la convicción tibia de las Naciones Unidas - un malestar en la sociedad (PNUD, I998). Por su parte, el poscolonialismo y el decolonialismo se han transformado en el reducto de un marxismo epistemológico (Grosfoguel, 2007) cargado de nativismo metodológico, al que Marx seguramente habría catalogado de socialismo utópico.

De manera alternativa a la mayoría de estas teorías, en este artículo nos interesa combinar dos elementos que generalmente han estado ausentes en las interpretaciones de la región latinoamericana del siglo XX antes mencionadas. Por un lado, un componente evolutivo e histórico entendido en el sentido de prácticas que producen eventos, cuya concatenación asociativa en términos de redes complejas puede conducir a transformaciones evolutivas o transiciones históricas (Sewell, 2005; Pombeni, 2016) y, por otro, un sentido sociológico sustentado en el principio de la diferenciación funcional como forma de organización social predominante en el actual momento evolutivo de la sociedad mundial (Luhmann, 20I2). En este sentido, buscamos ofrecer lineamientos para una sociología evolutiva de la diferenciación funcional en la región latinoamericana. En este sentido, nos alineamos con esfuerzos desarrollados previamente en el marco de una teoría sistémica de América Latina (Neves, I992; Mascareño, 20I0, 20I2; Cadenas, 20I2a, 20I2b). Nuestra hipótesis ahora es que la diferenciación funcional se desarrolla en América en coevolución con el proceso de diferenciación funcional en otras regiones de la sociedad mundial. ${ }^{2}$ Es decir, en el momento en que se produce el encuentro entre la sociedad en América y la sociedad europea, esto es, en el siglo XVI, ninguna de las dos sociedades regionales tiene predominio de la diferenciación funcional, sino de la 
estratificación. Por tanto, la diferenciación funcional no llegó con la empresa de conquista, sino que tuvo que desarrollarse - en su forma particular en América - en conexión con las prácticas sociales desplegadas en conjunto por ambas poblaciones. La modernización de América - y en particular de América Latina - no es, así, una alienación o un asesinato epistemológico, como las teorías de la identidad y las teorías decoloniales argumentan; tampoco es una historia que tenga un fin necesario en la revolución o una donde el pueblo sea, como un todo, objeto de explotación y la burguesía una arquetípica clase dominante - ya la historia del populismo latinoamericano invierte esta mirada. Del mismo modo, tampoco la evolución de América puede reducirse a etapas de desarrollo o estados de modernización; esta sería una aproximación muy restringida, limitada a la observación político-económica de un proceso evolutivo que no puede si no abarcar a la sociedad como un todo.

Para desplegar este argumento, dividimos esta aproximación en dos partes fundamentales. En la primera sección damos cuenta de los elementos centrales de una teoría de la diferenciación funcional de las sociedades modernas. Para esto nos basamos principalmente en la teoría de sistemas sociales. En la segunda sección ofrecemos los lineamientos principales de la evolución de la diferenciación funcional en América. Finalmente, ofrecemos algunas conclusiones de lo expuesto y perspectivas de trabajo futuro.

\section{LA DIFERENCIACIÓN FUNCIONAL LUHMANNIANA Y SUS ALTERNATIVAS}

La teoría de la diferenciación funcional es el aporte más relevante de Niklas Luhmann (20I2) para la sociología de la modernidad. Si bien se la puede relacionar nominalmente con la antigua teoría sociológica organicista de la diferenciación iniciada por Comte - replanteada en varios aspectos luego por Spencer, Durkheim y finalmente por Parsons, donde lo social se entiende como análogo a lo biológico - se trata de una propuesta radicalmente distinta a las de sus predecesores. Luhmann reformula estos conceptos para una teoría sistémica de la modernidad que no precisa abandonar todo lo relacionado con el organicismo biológico, pero que inevitablemente le quita protagonismo dentro de un entramado conceptual que recibe aportes de diversas disciplinas y formas de entendimiento.

Diferenciación funcional significa que no hay partes ni todo del cual se distingan, sino una constante reproducción de un sistema social en sí mismo. Cada subsistema diferenciado es el sistema total visto desde su propia perspectiva, por tanto, el sistema total no es una unidad descompuesta en partes, sino un horizonte compartido para cada sistema. Esto vale para todas las formas evolutivas que adquiere la diferenciación: segmentación, estratificación, centro/ periferia y diferenciación funcional. La función es un esquema de atribución causal en problemas definidos por cada subsistema y sus soluciones asociadas - con lo cual se reemplaza algún tipo de necesidad de un todo que se deba mantener por la contingencia de soluciones propuestas. 
Evolutivamente la diferenciación funcional caracteriza la estructura de la sociedad moderna en al menos cuatro aspectos:

- Inclusión universalista en sistemas funcionales y exclusión particularista por medio de organizaciones. Cada sistema funcional supone un horizonte social abierto a todos (para enfermarse, demandar derechos, participar políticamente, informarse, pagar), pero sin garantizar la participación efectiva que queda en manos de organizaciones.

- Codificación de todos los eventos posibles del mundo en valores binarios en cada sistema funcional (pagar/no pagar, conforme derecho/no conforme a derecho, enfermo/no enfermo). Se trata de esquemas de observación que destacan un lado de estos códigos en cada sistema y en los cuales los valores negativos no representan opuestos exclusivos al valor positivo, sino que operan como entorno indistinto en cada selección de un valor.

- Generalización social de problemas autoproducidos por los sistemas funcionales a lo ancho de la sociedad y medios simbólicos para probabilizar la aceptación de estos problemas y sus soluciones.

- Asincronía operacional de cada sistema social respecto de su entorno. Sistemas funcionales que definen internamente horizontes temporales de operación de manera independiente a la temporalidad definida en otros sistemas del entorno, sean estos otros sistemas funcionales, organizaciones o interacciones.

Modernidad es sinónimo de la emergencia de este nuevo tipo de diferenciación en un medio hasta entonces dominado por formas como la segmentación, la estratificación y centro/periferia. La diferenciación funcional no reemplaza, desplaza o suprime las formas anteriores, sino que esta se despliega paulatina y heterogéneamente, facilitando u obstaculizando la operación de las formas preexistentes. Es infructuoso sostener la hegemonía absoluta de la diferenciación funcional por sobre las demás formas, ya sea en América o en cualquier región del mundo.

La diferenciación funcional significa problemas importantes para segmentos, centros, periferias y estratos respecto de los esquemas de igualdad e inclusión establecidos. Por un lado, la segmentación logra adaptarse en parte al código de inclusión particularista de la estratificación, como familias o agrupaciones que se reconocen como totalidades iguales, pero en un entorno que reconoce y legitima las desigualdades. En los espacios de inclusión moderna universalista, por su parte, proliferan las indefiniciones para los segmentos como puede verse en las cuestiones de género o envejecimiento, o en las relaciones políticas entre Estados - mientras que las organizaciones se conducen con fluidez buscando oportunidades en un entorno segmentado. 
La estratificación, por su parte, se ajusta a la diferenciación funcional gracias a que hay estratos que logran reproducirse parasitariamente en organizaciones, pero sus fronteras están permanentemente amenazadas, ya sea por la propia dinámica funcional o por cuestiones más domésticas como las dificultades para el control de las alianzas matrimoniales. El enorme esfuerzo que significa crear y mantener la diferenciación estratificada hace que su representatividad se vea dramáticamente reducida. En suma, por una parte, la diferenciación funcional es una pesada carga para las formas anteriores y estas son obstáculos para el despliegue de la primera; pero, por otra parte, en las zonas de interpenetración entre formas hay también simbiosis bien arraigadas, como convertibilidades de medios y espacios de libertad para variaciones evolutivas.

Luhmann (2012) estudió prolíficamente la diferenciación funcional europea y su repercusión mundial, destacando por sobre todo los modos en que esta alcanzó un primado social estructural en ámbitos como la economía, la política, el derecho, la ciencia, el arte, la religión, los medios de masas, a la par de la diferenciación entre sistemas organizacionales e interacciones en un plano distinto. Entre las innovaciones más revolucionarias, la diferenciación funcional se entremezcla con referencias particularistas a personas, grupos, familias o territorios y las mueve hacia un universalismo abstracto e incluyente. La consecuencia directa es la formación de una sociedad mundial.

Evolutivamente, la emergencia de la diferenciación funcional fue un resultado más bien casual de eventos aleatorios que produjeron desviaciones morfogenéticas acumulativas en las estructuras y semánticas sociales de los siglos XII a XV especialmente en Europa, pero también en América. Tales desviaciones acumularon presiones en tantos ámbitos que se vieron finalmente desbordadas paulatina y escaladamente en sus capacidades. La diferenciación funcional no es entonces sinónimo de mejoramiento o bienestar, sino una forma plagada de riesgos y paradojas (Luhmann, 2012).

La cuestión acerca del alcance mundial de estas transformaciones requiere algunas explicaciones adicionales. En primer lugar, está lo que podemos llamar efecto ambiental. La nueva forma de diferenciación significó un cambio en el entorno en otras regiones, una variación posible de selección para abordar sus propios problemas internos siguiendo o traduciendo el modo foráneo. En segundo lugar, está el proceso endógeno; la diferenciación funcional como respuesta a problemas estructurales y semánticos análogos o equivalentes a los observados en la versión europea, con sus también semejantes efectos multidimensionales.

En cualquiera de los dos casos, no sin dificultad se estabilizaron las nuevas formas, mientras que hubo ámbitos que no se diferenciaron funcionalmente. Ni siquiera Europa, observa Luhmann (20I2), atestiguó la generalización social de ciertos códigos como la moral o la estabilización de funciones para 
sistemas como la educación; mientras en el entorno despuntaba la diferenciación funcional jurídica, artística, religiosa, científica, económica o política. Con esto, debiese quedar claro que la diferenciación funcional no es una forma perfecta y como proceso permanece inacabado.

A pesar de esto, la sociología latinoamericana, como hemos visto previamente, ha gustado de observar su modernidad como una versión incompleta de un supuesto modelo europeo original y se ha ubicado a sí misma en el lado desfavorecido de su propia codificación del mundo; como una teoría de dependencia, subdesarrollo, subordinación y, hoy especialmente, colonización. Una diferenciación funcional realizada a medias o simplemente impuesta parcialmente por la fuerza. Pero si, como hemos destacado, la diferenciación funcional se reconoce dentro de un marco evolutivo que destaca transformaciones y no reniega de formas que pueden ser vistas como incompletas, imperfectas o movilizadas por cuestiones internas y externas, el punto de comparación, entonces, se desvanece o a lo menos se pierde de vista.

Lo que quisiéramos desarrollar a continuación es una alternativa a la tendencia general. Sostendremos que la diferenciación funcional latinoamericana no es una deformación de un plan original - pues dicho plan nunca tuvo lugar - sino que se describe de mejor modo como una trayectoria de la diferenciación funcional que, como la europea y relacionada con ella, posee lados claros y oscuros. Reconocemos así los efectos empíricos mundiales de esta forma de diferenciación y las presiones que ejerce, pero atendemos también a los plexos que produce empíricamente en regiones del mundo como la latinoamericana que posee, por su parte, su propia trayectoria estructural y semántica, y que ha puesto en marcha a su manera sus transformaciones.

\section{LA TRAYECTORIA LATINOAMERICANA DE LA DIFERENCIACIÓN FUNCIONAL}

El concepto de trayectoria de la diferenciación funcional dialoga con teorías como la de las modernidades múltiples (Eisenstadt, 2000) y la sociedad mundial (Stichweh, 2000). Basándose en categorías como rutas hacia y a través de la modernidad, Göran Therborn (I996) ha sostenido la tesis de las modernidades entrelazadas [entangled modernities], destacando la presencia de redes de comunicación y transferencias simbólicas no lineales en la constitución de la sociedad mundial. Esta idea es de particular importancia, pues con ella se puede apoyar la objeción de que la diferenciación funcional ha sido un orden inducido - si no impuesto - en América o el resto del mundo.

Stichweh (2013) ha sostenido que hay tres mecanismos fundamentales para la constitución de la sociedad mundial: difusión, descentralización e interrelación de sistemas funcionales. Mientras los dos primeros conducen a la tradicional visión de la modernidad que hemos criticado previamente, el último permite, en cambio, una perspectiva distinta. Si nos centramos en la difusión, 
el diagnóstico comparativo será el de las desviaciones y corrupciones del modelo original; al atender a la descentralización, por su parte, la mirada se dirigirá hacia la diáspora de instituciones europeas y los diversos significados asociados a ellas. Si, en cambio, enfatizamos en las interrelaciones, nos dirigiremos hacia concepciones relacionales y no hacia un modelo original como estándar evaluativo.

De este modo, la hibridación estructural y semántica es la clave para descifrar las múltiples trayectorias de la diferenciación funcional y entender el modo en que las clásicas promesas de la modernización - firmemente arraigadas en la autonomía de los sistemas funcionales (libertad de prensa, representatividad y participación democrática, mercados libres y Estado de derecho) - se organizan en diferentes combinaciones de expectativas en distintas regiones del mundo, sufren vaivenes significativos y se adaptan situacionalmente a estas variaciones. Pueden ser tanto selecciones evolutivas menores como planificaciones a gran escala las que amplifiquen cambios importantes, como muestran los procesos actuales de monetización en Chile, Argentina o Brasil, o de politización en Venezuela, Bolivia o Ecuador, con sus inestabilidades y resistencias (Kingstone, 20I8); así como los conservadores en Estados Unidos presionan por cerrar fronteras y restringir el comercio, y en parte de Europa crece la alternativa que ofrece la extrema derecha para gobernar con una democracia más bien mínima (Hedges, 20I9). No parece entonces paradojal que China se convierta en defensora tanto del libre mercado como de la censura informativa, o que Rusia sea a la vez aliado y enemigo de Occidente (Lukin, 20I8).

Como las demás trayectorias de la diferenciación funcional, la latinoamericana se puede caracterizar en periodos relativamente marcados por tendencias favorecidas por cuestiones internas y externas. Caracterizaremos estos periodos sumariamente como: pluralismo, centralización, Estados nación incluyentes y excluyentes, y redes incrustadas de inclusión y exclusión.

\section{PRIMER PERIODO: PLURALISMO}

La conquista de América fue una empresa sangrienta; no hay duda de ello. Se pueden discutir los números de muertes causadas por las guerras entre conquistadores y nativos o las epidemias desatadas en dicho periodo, pero el hecho fundamental sigue en pie. No obstante, si consideramos el proceso desde un punto de vista de sociología evolutiva, podemos apreciar interesantes correlaciones entre los momentos evolutivos de América y de Europa:

- En primer lugar, el corporativismo de la monarquía ibérica del siglo XV se ajustaba bastante bien al pluralismo étnico de América. Las altas culturas prehispánicas estaban organizadas en formas de diferenciación estratificadas, rodeadas de periferias laxamente acopladas y grupos seg- 
mentados. Los virreinatos fundados por los españoles presentaban una estructura similar: centros con instituciones comerciales (tribunales de consulados), legales (audiencias) y políticas (cabildos); y periferias organizadas en municipalidades (Mirow, 2004).

- En segundo lugar, municipalidades que eran prácticamente reinos en sí mismos. Estas pretendían el control de territorios sin eliminar necesariamente su diversidad. Si bien se relacionaban hacia arriba con las estructuras jerárquicas del gobierno colonial, también construían hacia abajo redes de cooperación relativamente independientes entre nativos, sus líderes y seguidores, con sus propias expectativas cognitivas y normativas (Tau, 2015).

- En tercer lugar, hubo un cierto espacio para la libertad y el pluralismo, no debido a fuertes ideas liberales puestas en práctica en América, sino por la imposibilidad estructural y semántica de ocupar todo el territorio (Adorno, 2008).

A partir de lo anterior se pueden establecer algunas consecuencias. Primero, no hubo un choque de formas de diferenciación entre América Latina y las monarquías europeas. No se trató de una sociedad funcionalmente diferenciada imponiendo sus estructuras a sociedades segmentarias, sino de un ajuste de formas equivalentes de diferenciación segmentada, estratificada y de centro/periferia. Segundo, si se acepta lo anterior, la diferenciación funcional no proviene exclusivamente de Europa, sino que también se desarrolla en América, en sus relaciones comerciales, legales, religiosas y políticas con y dentro de las colonias. Tercero, si la diferenciación funcional en América se desarrolla en relaciones, entonces las prácticas sociales americanas y las europeas tienen que producir eventos de ajuste mutuo que se conforman en redes de distintas escalas. Cuarto, las acciones de estas redes pueden institucionalizarse en sistemas sociales funcionalmente diferenciados o pueden hacerlo en formas multiescalares estratificadas que apoyan o disputan los rendimientos de las instituciones nacientes de la diferenciación funcional. Y quinto, si todo lo anterior es plausible, entonces un determinado tipo de pluralismo tuvo lugar en América en el encuentro con los ibéricos.

No se trató, por cierto, (no podía tratarse) de un pluralismo moderno en el cual las diversas formas adoptaron relaciones semi-igualitarias entre sí. Se trató aquí de una ficción de igualdad en el intercambio, hasta el momento en que hubo que ejercer el poder político por medio de los impuestos o la ocupación y desplazamiento físico de la población. El pluralismo del siglo XVI tiene que ver, por un lado, con formas jurídicas que reconocen autonomía a las colonias, no tanto por un principio moral, sino porque la extensión territorial es tan vasta que es imposible una ocupación de hecho. Por otro lado, ese pluralismo se asocia con la variedad de principios de diferenciación que se interrela- 
cionan de modos fuertes e igualitarios entre sí, tanto por el lado americano como por el lado europeo: existe un principio de segmentación (sociedades tribales, virreinatos, municipalidades), uno de centro y periferia (religiosa, económica y políticamente organizado, tanto por el lado americano como por el europeo; con una creencia con pretensión universal, impuestos y fuerzas de ataque y defensa en ambos casos) y un principio de estratificación (estamental por el lado americano, monárquica por el lado europeo). En ese marco de relaciones complejas comienza a tener lugar la diferenciación funcional para ambas sociedades regionales.

A este respecto, cabe mencionar algunos ejemplos de condensaciones tempranas de operaciones funcionales y de red en América Latina:

- En la economía, aparecen exaptaciones relacionadas con la necesidad de acuñar monedas a nivel local para recaudar impuestos a un nivel regional. El intercambio económico ya existía ciertamente antes en América, por ejemplo, usando el cacao como un medio análogo al dinero en distintos intercambios de red. Aparecen monedas acuñadas posteriormente en asentamientos mineros y para el comercio regional del siglo XVI (Bulmer-Thomas, 2003).

- Las relaciones financieras aparecen también en el siglo XVI, junto con los créditos entregados por las municipalidades a los nativos para la producción agraria (Bulmer-Thomas, 2003).

- En términos religiosos, las llamadas congregaciones de franciscanos, dominicanos y jesuitas concentran las funciones de evangelización. Las misiones jesuitas en particular resultan muy interesantes, pues tienden a preservar las estructuras y semánticas sociales tradicionales de los evangelizados mientras promueven paralelamente la cristiandad (González \& Pupo-Walker, 2008).

Sin embargo, el despegue más importante de la religión en América Latina es la religiosidad popular. La falta de clérigos, la reproducción de creencias como forma de resistencia a los poderes coloniales y el hecho que la mayoría de los clérigos prefirieran trabajar con europeos crearon un vívido espacio para la comunicación de la religiosidad popular entrelazada complejamente con la cristiandad del siglo XVI.

- En lo jurídico, los "indios" - y todas las denominaciones semejantes dadas a una pluralidad de grupos étnicos nativos - fueron incluidos en el estatus de personas miserables, como viudas, huérfanos y gente rural. Se les consideró como vasallos de la corona, pero libres y dicha libertad - reconocida tempranamente en el I500 - fue la base para preservar sus propias costumbres y leyes. De este modo, el sistema legal americano 
se desarrolló originalmente más como un pluralismo legal que una estructura centralizada (Duve \& Pihlajamäki, 20I5).

- En lo político, como señalamos previamente, los virreinatos y municipalidades establecieron la infraestructura para el funcionamiento de las relaciones jerárquicas de poder con las metrópolis, así como también redes con los locales (Bethell, I985).

Visto de este modo, no existe una forma mecánica de traslación de las prácticas de la diferenciación funcional desde Europa hacia América. No podía haberla, pues Europa no era una sociedad con predominio de la diferenciación funcional en el siglo XVI, sino con predominio de la estratificación. Incluso para Europa este es un siglo de apogeo de las formas renacentistas. Un siglo en el que se realizan múltiples avances en ciencia y política (Smith, I 982; 2007) que preparan el camino hacia el proceso de diferenciación funcional, especialmente durante del siglo XVII conocido en la historiografía europea como el siglo de la crisis (Parker, 2013; Trevor-Ropper, I999).

Visto desde la perspectiva americana, la situación no era muy distinta en términos evolutivos. Las sociedades maya y azteca se encontraban en su período posclásico con alto desarrollo científico y la centralización política de un Estado imperial centro-periférico y claramente estratificado solo comparable al imperio mesopotámico de la Antigüedad. América no estaba poblada "por unos cuantos indios". Incluso se renovaba evolutivamente en eventos catastróficos como la sobrepoblación maya del período clásico al posclásico (Culbert, 2003) o el incendio de la ciudad de Teotihuacán como protesta ante la opulencia de los centros, que concluye con el período clásico y da inicio al posclásico propiamente azteca (Millon, 2003).

Cuando las cosas se plantean de este modo no es fácil aceptar que, con baratijas y espejos más una supuesta racionalidad instrumental superior, los europeos dominaron y subyugaron a los americanos; más aún si se piensa que los europeos que llegaban inicialmente a América no eran necesariamente los más dotados racionalmente (Saldaña \& Madrigal, 2007).

\section{SEGUNDO PERIODO: CENTRALIZACIÓN}

El pluralismo americano se desarrolló a lo largo de dos siglos hasta las reformas borbónicas del siglo XVIII. El objetivo era concentrar las tendencias centrífugas hacia una estructura burocrática jerárquica que tenía a España como el centro y a América en la periferia (Weber, 2004). Con ello, el proceso de diferenciación funcional comienza a sufrir un contraproceso de centralización, especialmente alrededor del poder administrativo del Estado.

Esto no es solo una particularidad del Imperio Español. El colonialismo en general (el inglés, el francés) operó mediante una pretensión de control de la diferenciación funcional que había sido impulsada, en diversas regiones, por 
el propio colonialismo. El intento de control tomaba modelos distintos: un pluralismo jurídico en el caso español, una inclusión política y simbólico-monárquica en el caso inglés de la Commonwealth, y un modelo republicano estatal en el caso francés (cuyos mejores ejemplos son Argelia y Córcega).

En cualquier caso, los tres modelos son apoyados por las armas. Su importancia la entendían bien las reformas borbónicas. Mediante ellas se podía contrarrestar la descentralización y fragmentación del poder que producían las redes, en el marco plural de formación de la diferenciación funcional en los primeros dos siglos del proceso de coevolución, entre la región americana y la europea. Así, en el caso americano, las medidas borbónicas incluyeron, entre otras cosas:

- Organización centralizada de una fuerza militar para defender las posiciones ganadas frente a otros poderes coloniales y para avanzar en la ocupación del territorio.

- Expulsión de los jesuitas de América para poder concentrar las designaciones educativas y religiosas.

- Introducción de la "libertad de comercio" con la Corona para disolver las economías locales y corporaciones.

- Organización de la economía mediante un sistema de impuestos centralizado.

- Ampliación de las audiencias, excluyendo a los criollos de su participación (Véliz, I98I).

Hay un relativo consenso entre los historiadores en que a las reformas borbónicas les faltó coherencia y efectividad (Mirow, 2004). Estas reformas favorecieron a las elites españolas, pero alienaron a los criollos y a los nativos acostumbrados a las expectativas de un funcionamiento descentralizado de las estructuras corporativas. El creciente antagonismo entre las elites españolas y los poderes locales condujo a las guerras de independencia a inicios del siglo XIX y, con ello, a la construcción de los Estados nacionales en América Latina.

\section{TERCER PERIODO: ESTADOS NACIONALES INCLUYENTES Y EXCLUYENTES}

Las guerras de independencia en América Latina fueron más bien producto de las pugnas por controlar el Estado, entre los poderes regionales organizados en redes y las metrópolis europeas, que de las reformas borbónicas (Dawson, 2010). En este sentido, el proceso de construcción de los Estados prestó más interés a la concentración de poder político y recursos económicos que a establecer instituciones de bienestar. Una ciudadanía más bien formal, expresada en constituciones y discursos de inclusión política, contrastaba con una limitada experiencia de inclusión institucional.

La construcción elitista de la experiencia institucional fue terreno fértil para el crecimiento de las redes en dos direcciones. Por un lado, procurando 
asegurar posiciones privilegiadas en la política y en la emergente economía de exportaciones. Por el otro, fomentando la inclusión política y económica por medio de redes con caudillos locales y a través de las haciendas (Carmagnani, 2004).

El Estado-nación se convirtió así en un Estado multinivel. Mientras los niveles superiores estaban destinados solamente a terratenientes, empresarios y a la primera generación de clases medias educadas, los inferiores incluían "indígenas", campesinos, sirvientes, inquilinos y a un emergente proletariado urbano surgido de la economía de exportaciones. Este periodo ha sido llamado modernización oligárquica (Larraín, 2004). En los inicios del siglo XX, las capas inferiores fueron incluidas semánticamente como pueblo (Mascareño, 20I 2)

La más importante consecuencia de las reformas borbónicas, especialmente para la construcción de los Estados nacionales latinoamericanos, fue una marcada centralización en la operación de sistemas funcionales que ya se había venido anticipando desde las reformas borbónicas. Esta tendencia centralizadora tiene varias expresiones codificadas:

- Espacialmente, introduce la distinción entre áreas metropolitanas y periferias.

- Estructuralmente, favorece la toma de decisiones centralizada, excluyendo los nodos locales de participación política.

- Socialmente, produce diferentes redes multiescalares, ya sea para reproducir secuencias de inclusión o para presionar por inclusión en los centros.

- Semánticamente, se distingue entre civilización en centros urbanos y barbarie en las periferias.

- Normativamente, se promueven valores europeos, más específicamente valores de carácter universalista y de origen francés, como vía exclusiva para convertirse en sociedades modernas.

La centralización, no obstante, no elimina la pluralidad estructural desarrollada en América a lo largo de dos siglos, sino que la transforma en redes que se disputan rendimientos operacionales.

\section{CUARTO PERIODO: REDES ANCLADAS DE INCLUSIÓN Y EXCLUSIÓN}

Como lo hemos presentado en la introducción de este artículo, existen varias teorías sociológicas relativas a la trayectoria de América Latina, especialmente en los siglos XIX y XX. Por ejemplo, la teoría del desarrollismo de la CEPAL de fines de los años I 940 que distinguió, desde un punto de vista económico, regiones desarrolladas funcionalmente diferenciadas de zonas subdesarrolladas (Larraín, 2004); la teoría de la modernización de Gino Germani (I98I) que analizó la asincronía entre un polo moderno, funcionalmente diferenciado, y otro 
tradicional; la teoría latinoamericana de la dependencia que reintrodujo la distinción centro/periferia en la periferia, enfatizando así la dominación y explotación de clase en zonas subdesarrolladas, adoptando un punto de vista más político (Cardoso \& Faleto, I990); la versión católica-esencialista de la identidad latinoamericana, para la cual la reflexividad de la diferenciación funcional encubre el sustrato pre reflexivo genuinamente católico de amor y comunalidad heredado de la España colonizadora (Morandé, I987); la teoría de la modernidad periférica de Marcelo Neves (I992), en la cual la autonomía sistémica parece ser más bien una experiencia excepcional en América Latina; y el argumento poscolonial que busca recuperar prácticas y símbolos precoloniales, y enfatizando el derecho a la coexistencia epistemológica de las diferencias, tal como sostiene el enfoque de Walter Mignolo (2005).

Frente a estas interpretaciones, no buscamos encontrar el justo medio. Basta con señalar que la diferenciación funcional contemporánea en América Latina se presenta bajo presiones constantes de redes estratificadas multiescalares que actúan como atractores extraños para las operaciones funcionales, produciendo así bifurcaciones críticas de inclusiones y exclusiones respecto de los rendimientos sistémicos. Dichas redes han coevolucionado con la diferenciación funcional y no deben ser concebidas como meras prácticas tradicionales, estructuras subdesarrolladas, o simples formas parasitarias de organización social - por supuesto, tampoco como fuente de la verdadera identidad latinoamericana.

Estas redes estratificadas son formas más bien modernas de organización social que presentan diferentes posibilidades de conectividad en cada una de sus escalas (Hoevel \& Mascareño, 20I6). Ellas crean ejes de acumulación, distribución y asignación de recursos que pueden sobrepasar marcos sistémicos, infiltrar sus barreras de convertibilidad y producir bifurcaciones inesperadas de inclusiones y exclusiones (Cadenas, 2012a).

No obstante, lo anterior no significa que en la región latinoamericana no tengan lugar medios simbólicos diferenciados, operaciones autónomas, acoplamientos estructurales relativamente estabilizados e interdependencias funcionales. Más bien significa que las interrelaciones entre escalas de las redes ofrecen diferentes modos de inclusión y justificaciones para la exclusión en múltiples situaciones sociales.

En algunos casos estas redes conllevan efectos acumulativos de exclusiones e ilegalidades. Conocidos son los casos de corrupción política de Lava Jato en Brasil o de Soquimich en Chile; la distorsión de precios descubierta en las colusiones entre grandes empresas del retail en Colombia y también Chile; la estructuración de partidos políticos con base en redes multiescalares de inclusiones en el caso del peronismo argentino investigado por Javier Auyero (2000, 20I2); las redes de favores entre policías y delincuentes, también en Argentina, analizadas por Matias Dewey (20I I); los llamados tribunales crimina- 
les compuestos de miembros de las más grande red delictiva de São Paulo, en Brasil (Feltran, 20Io); los grandes cárteles de droga en Colombia o México; o la cartelización del Estado venezolano desde 2000 en adelante (Altez, 20I9). La lista podría ampliarse significativamente.

Estas redes también pueden operar como mecanismos de inclusión particularista con efectos menos dramáticos, como las redes de apoyo entre la población más pobre y los servicios públicos de asistencia - promovidas incluso como políticas públicas que reconocen estas estructuras como una oportunidad a explotar; en las redes de contactos y conocidos que mueven oportunidades de empleo y privilegios en burocracias estatales o empresas privadas pero que no son autosuficientes, en tanto se demanda un mínimo de competencias; o en los propios entrelazamientos que organizaciones establecen para fortalecer la posición de intereses gremiales o de otro tipo en entornos de competitividad.

En síntesis, en primer término, la trayectoria latinoamericana de la diferenciación funcional ofrece un amplio rango de posibilidades actuales para la adaptación de expectativas cognitivas. Se puede aprender que determinadas acciones ilegales operan como mecanismos de inclusión cuando las alternativas procedimentales no lo hacen. También la justificación para cambios de regímenes políticos se vuelve un argumento moral: "puesto que la sociedad excluye a los pobres, ellos estarían en su derecho de actuar distinto"; o el caso de los políticos: "nuestros opositores son ricos, de modo que se justifica recibir dinero de las empresas que regulamos para equilibrar la representatividad política". Estos no son problemas exclusivos de estructuras normativas débiles, sino de equilibrios múltiples para reducir la brecha entre expectativas y vivencias actuales.

Segundo, desde el punto de vista de las estructuras normativas, las expectativas muestran más bien una deflación. Como señaló Parsons (I968), la deflación de los compromisos de valor se produce por expectativas incumplidas. Como consecuencia, la unidad en deflación busca compromisos reales y castiga duramente el incumplimiento. En otras palabras, mientras más deflación sufren los compromisos de valor, más violentos serán los conflictos y reacciones que cabe esperar. Esto podría explicar en parte la permanente inestabilidad política de la región y la prevalencia de la violencia como modo de resolución de conflictos.

Tercero, en lugar de zonas de inclusión y exclusión, en América Latina se presentan redes ancladas en relaciones funcionales que acumulan, distribuyen y asignan recursos materiales y simbólicos provistos por sistemas funcionales. Como hemos visto previamente, estas redes pueden tomar control de las barreras de convertibilidad entre sistemas y transformarse en porteros activos de rendimientos sistémicos. 
Cuarto, como ha señalado Stichweh (2000), la exclusión en distintas regiones de la sociedad mundial se relaciona con el debilitamiento de la función de promoción de la inclusión por parte de los Estados de bienestar. Este último intercede cuando los sistemas funcionales estabilizan o refuerzan las exclusiones. Lo anterior es doblemente cierto para América Latina. Por un lado, los Estados latinoamericanos han tenido siempre problemas para controlar la acumulación de recursos materiales y simbólicos, produciendo una especie de sobreinclusión de grupos y redes en los escalones superiores, como en el caso de las elites. Las elites controlan entonces el Estado y los problemas de acumulación permanecen sin solución. Por otro lado, la extensión estructural, la profundidad histórica y complejidad semántica de las exclusiones en América Latina sobrepasan las capacidades de los Estados, y cuando esto ocurre, se abre un espacio a las redes estratificadas para cumplir o suplir la función de promoción de la inclusión del Estado de bienestar - se trata de redes con expectativas similares, pero con una concepción diferente de la legalidad y con justificaciones distintas a las de los Estados de bienestar para la inclusión y la exclusión social.

\section{CONCLUSIONES}

En este artículo hemos desplegado dos argumentos centrales interrelacionados. El primero indica que el modo predominante de organización social de la sociedad mundial, la diferenciación funcional, no ha sido una imposición de la sociedad europea sobre la americana, pues en el momento del encuentro de ambas sociedades regionales a fines del siglo XV, tanto en América como en Europa existe un predominio de la estratificación combinada con formas fuertes de segmentación y de organización centro-periférica. La consecuencia de este argumento es que la diferenciación funcional tuvo que desarrollarse en la interrelación de ambas sociedades regionales, por lo que ella no es un fenómeno 'más europeo' de lo que es americano.

El segundo argumento indica que la pluralidad de principios de organización social que tiene lugar en el tránsito del siglo XV al siglo XVI, hace que las redes de interacción adquieran en América una presencia fundamental para la construcción del orden social. Esto es visible desde la fase original pluralista hasta el modelo actual de institucionalización de redes multiescalares e instituciones de la diferenciación funcional con pretensión de autonomía operativa. De este modo, no se debe entender las redes como una forma necesariamente antagónica a las instituciones de la diferenciación funcional; ambas se apoyan y se disputan mutuamente los recursos (sociales, naturales, simbólicos) para la reproducción de su propia lógica y, por cierto, para la reproducción de su propia integración.

Puesto que la mayor parte de las teorías sociológicas sobre América la observan y analizan, en el mejor de los casos, desde el período de independen- 
cia de los Estados nacionales (esto es, desde unas décadas antes de que comience a denominarse América Latina), no pueden observar ninguno de los hechos que sintetizamos en los dos argumentos anteriores. Estas teorías asumen que la sociedad americana nace a la vida global cuando en ella surge el Estado moderno, lo que muestra su claro nacionalismo metodológico como principio de investigación social. Por otro lado, las teorías que logran desprenderse de este principio analítico y van a las raíces de la modernidad americana, como la de Pedro Morandé (I987), consideran que, al alcanzar la diferenciación funcional un cierto predominio en la organización de la región, este constituye una alienación de las "verdaderas" formas de organización que tenían lugar hace ya aproximadamente 530 años, con lo que quedan en una misma posición que las teorías poscoloniales o decoloniales, para las que cerrar la herida colonial significa nada menos que eliminar la evolución.

Por el contrario, nuestras argumentaciones se apoyan en la evolución para proponer, en un plano metodológico, lo que queremos denominar sociología evolutiva. En ciertos lugares Niklas Luhmann ha sostenido que la diferenciación funcional es el principio de diferenciación predominante en la sociedad mundial contemporánea; en otros se ha hecho la pregunta si la diferenciación funcional no es más bien una anomalía europea. Una sociología evolutiva debiera poder esclarecer este problema. En lo que hemos sostenido en este artículo, para el caso de América y Europa en el tránsito del siglo XV al XVI, la diferenciación funcional emerge en relaciones económicas, jurídicas, políticas, religiosas, educativas, y muchas más entre distintas regiones del mundo.

El caso del intercambio entre América y Europa puede ser particular; más similar a la relación entre Asia y Europa que, por ejemplo, a la relación entre África y Europa. La diferencia es que, al momento del contacto, la sociedad africana se organiza predominantemente por un principio de segmentación antes que por centros y periferias o por estratificación. Asia, América y Europa son evolutivamente similares hacia los siglos XV y XVI, lo que hace que puedan reconocerse mutuamente en cuanto a formas de orden social y emprender un camino conjunto en la formación evolutiva de la diferenciación funcional. En cambio, la particularidad africana de mantener un principio predominante de segmentación hasta entrado el siglo XIX hace imposible la participación común, salvo como suplemento en el comercio global de esclavos y en la explotación extractivista indiscriminada de recursos naturales sin oposición, como sí acontece en el caso americano - aun cuando no en el asiático por un desarrollo hipertrófico de la administración del Estado como centro del sistema político (en otras palabras, gobiernos autocráticos o totalitarios).

Una sociología evolutiva es una sociología de la formación global de la sociedad mundial. En ese sentido, el componente histórico es fundamental. Pero, al contrario de la historia, una sociología evolutiva no analiza períodos aislados, sino que siempre debe tener en perspectiva la transición de un mo- 
mento evolutivo a otro; debe considerar los eventos históricos y las prácticas sociales como elementos de un orden mayor en el cual se entrecruzan de maneras complejas diversos principios de diferenciación social. Asimismo, una sociología evolutiva no puede hacerse regionalmente, como si la evolución social pudiese formarse endógenamente en espacios aislados. Una sociología evolutiva es sociología evolutiva de la sociedad mundial. Solo de ese modo se pueden observar las diferencias y similitudes que dan pie a la consolidación o al colapso de ciertas formaciones sociales, a su superación o a su permanencia dinámica en un horizonte temporal indefinido. Por ello, analizar la diferenciación funcional en América es también analizarla en su vínculo con Europa; tal como observar la europea debe ser ponerla en relación con otras regiones de la sociedad mundial, como la región americana.

La sociología en América Latina ha llegado, a lo más, a entender que la historia es un elemento relevante de la comprensión científico social del presente. Esta carta pueden jugarla tanto el marxismo como las teorías de la modernización; las teorías de la identidad tanto como el desarrollismo. Sin embargo, porque esas teorías no alcanzan el nivel de abstracción para comprender el horizonte evolutivo en el que la historia tiene lugar, no pueden darse cuenta de la originalidad de los procesos americanos en su relación con Europa durante los últimos cinco siglos y medio. De ahí que hayan propagado la impresión de que la sociedad americana es un reflejo de la sociedad europea o un intento de emulación fallido de sus formas de organización social, una incompletitud o una ausencia. Por el contrario, en este artículo hemos sostenido que la historia de la diferenciación funcional en la sociedad mundial surgió, al menos, en una interrelación entre Europa y América. Investigaciones futuras, y probablemente desde otras regiones, deberán analizar si esto es una particularidad del vínculo entre Europa y América o si la descentralización de la evolución es el estándar de la sociedad mundial. 
Hugo Cadenas es doctor en sociología por la Universidad Ludwig Maximilian de Múnich, Alemania. Profesor de los departamentos de Antropología y Trabajo Social de la Universidad de Chile. Sus áreas de interés son la teoria sociológica y antropológica, sociologia del derecho, desigualdad social, ética y medioambiente. Entre sus últimas publicaciones se cuentan: "Sistemas y sistema mundo: la crisis teórica en Immanuel Wallerstein" (Estudios Públicos, 20I9); "Un modelo conceptual para los comités de ética: síntesis sociológicas preliminares" (Acta Bioethica, 20I9); "Radcliffe-Brown, Alfred R. (I88I-I955)" (The Blackwell Encyclopedia of Sociology, 2019).

Aldo Mascareño es doctor en sociología por la Universidad de Bielefeld, Alemania. Investigador senior del Centro de Estudios Públicos y profesor de la Universidad Adolfo Ibáñez, Chile. Sus áreas de interés son la teoria sociológica, sociologia del derecho, derecho mundial y teorías de la complejidad. Entre sus últimas publicaciones se cuentan: "Ética de la contingencia para mundos incompletos" (Diferencias, 2019); "Constituent crises. The power of contingency" (Revista Brasileira de Sociologia do Direito, 20I9); "La crisis como control de hipertrofia" (Revista Direito Mackenzie, 2018). 


\section{NOTAS}

I Los autores agradecen los valiosos comentarios y sugerencias de los revisores anónimos de la revista al artículo.

2 Hablamos aquí de "América" para identificar la región de América Latina en el período previo a la generalización de su designación como "América Latina" en el tránsito del siglo XIX al XX.

\section{REFERENCIAS BIBLIOGRÁFICAS}

Adorno, Rolena. (2008). Cultures in contact: Mesoamerica, the Andes, and the European written tradition. In: Gonzalez, Roberto \& Pupo-Walker, Enrique (eds.). The Cambridge History of Latin American Literature. Cambridge: Cambridge University Press, p. 33-57.

Altez, Rogelio. (2019). Poder, negocios y destrucción. Los apagones de Venezuela en marzo de 2019 y la cartelización del Estado. Estudios Públicos, I56.

Auyero, Javier. (2012). Poor people's lives and politics: the things a political ethnographer knows (and doesn't know) after I5 years of fieldwork. New Perspectives on Turkey, 46, p. 95-I 27.

Auyero, Javier. (2000). The logic of clientelism in Latin America: an ethnographic approach. Latin American Research Review, 35/3, p. 55-8I.

Bethell, Leslie. (1985). Colonial Latin America. The Cambridge History of Latin America. Cambridge: Cambridge University Press.

Bulmer-Thomas, Victor. (2003). The economic history of Latin America since independence. Cambridge: Cambridge University Press.

Cadenas, Hugo. (2012a). La desigualdad de la sociedad. Diferenciación y desigualdad en la sociedad moderna. Persona y sociedad, 26/2, p. 5I-77.

Cadenas, Hugo. (20I2b). Algunas tendencias de la diferenciación del derecho en Chile. Revista de Ciências Sociais, 2/I, p. 72-I07.

Cardoso, Fernando Henrique \& Faletto, Enzo. (I990). Dependencia y desarrollo en América Latina. Ciudad de México: Siglo XXI. 
Carmagnani, Marcello. (2004). El otro Occidente. Ciudad de México: Fondo de Cultura Económica.

Culbert, Thomas P. (2003). The collapse of classic Maya civilization. In: Yoffee, Norman \& Cowgill, George (eds.). The collapse of ancient states and civilizations. Tucson: The University of Arizona Press, p. 69-Ior.

Dawson, Alexander. (2010). Latin America since independence: a history with primary sources. London: Routledge.

Dewey, Matías. (20II). Fragile States, robust structures: illegal police protection in Buenos Aires. GIGA Working Papers, I69.

Duve, Thomas \& Pihlajamaki, Heikki (eds.). (20I5). New horizons in Spanish colonial law. Frankfurt: Max Planck Institute for European Legal History.

Eisenstadt, Shmuel. (2000). Multiple modernities. Daedalus, I29/I, p. I-29.

Fajnzylber, Fernando. (1983). La industrialización trunca de América Latina. Ciudad de México: Editorial Nueva Imagen. Feltran, Gabriel. (20I0). Crime e castigo na cidade: os repertórios da justiça e a questão do homicídio nas periferias de São Paulo. Caderno CRH, 23/58, p. 59-73.

Germani, Gino. (I98I). The sociology of modernization. New Brunswick/London: Transaction Books.

Germani, Gino. (1962). Política y sociedad en una época en transición. Buenos Aires: Paidós.

Gonzalez, Roberto \& Pupo-Walker, Enrique (eds.). (2008). The Cambridge history of Latin American literature. Cambridge: Cambridge University Press.

Grosfoguel, Ramón. (2007). The epistemic decolonial turn. Beyond political-economy paradigms. Cultural Studies, 2I/2-3, P. 2II-223.

Hedges, Chris. (2019). America: The farewell tour. New York: Simon \& Schuster, Inc.

Hoevel, Carlos \& Mascareño, Aldo. (20I6). La emergencia de redes clientelares en América Latina: una perspectiva teórica. MAD, 34, p. 36-64.

Jaguaribe, Hélio. (1976). Crises e alternativas da América Latina. São Paulo: Perspectiva. 
Kingstone, Peter. (20I8). The political economy of Latin America. New York/London: Routledge.

Larraín, Jorge. (2004). Identidad y modernidad en América Latina. Ciudad de México: Océano.

Luhmann, Niklas. (2012). Theory of society. Stanford: Stanford University Press.

Lukin, Alexander. (2018). China and Russia. The new rapprochement. Cambridge: Polity Press.

Mariátegui, José Carlos. (2007). Siete ensayos de interpretación de la realidad peruana. Caracas: Biblioteca Ayacucho.

Mascareño, Aldo. (2010). Diferenciación y contingencia en América Latina. Santiago: Ediciones Universidad Alberto Hurtado.

Mascareño, Aldo. (2012). Die Moderne Lateinamerikas. Bielefeld: transcript Verlag.

Mignolo, Walter. (2005). La idea de América Latina. Barcelona: Gedisa.

Millon, René. (2003). The last years of Teotihuacan dominance. In: Yoffee, Norman \& Cowgill, George (eds.). The collapse of ancient states and civilizations. Tucson: The University of Arizona Press, IO2-I64.

Mirow, Matthew C. (2004). Latin American Law. Austin: University of Texas Press.

Morandé, Pedro. (I987). Cultura y modernización en América Latina. Madrid: Ediciones Encuentro.

Neves, Marcelo. (1992). Verfassung und Recht in der peripheren Moderne - Eine theoretische Betrachtung und eine Interpretation des Falls Brasilien. Berlin: Duncker \& Humboldt.

Parker, Geoffrey. (2013). Global Crisis. War, climate change, and catastrophe in the seventeenth century. New Haven: Yale University Press.

Parsons, Talcott. (1968). On the concept of value-commitments. Social Inquiry, 38/2, p. I35-I60.

PNUD. (I998). Informe sobre el desarrollo humano Chile: las paradojas de la modernización. Santiago: PNUD.

Pombeni, Paolo. (2016). The historiography of transition. Abingdon: Routledge. 
Ribeiro, Darcy. (2008). O povo brasileiro. São Paulo: Companhia de Bolso.

Saldaña, Juan Jose \& Madrigal, Bernabe. (2007). Science in Latin America: a history. Austin: University of Texas Press. Sewell, William. (2005). Logics of history. Chicago: University of Chicago Press.

Smith, Adam. (2007). The wealth of nations. Hampshire: Harriman House Ltd.

Smith, Adam. (1982) The history of astronomy. In: Smith, Adam. Essays on philosophical subjects. Illinois: Liberty Fund, Inc., p. 33-105.

Stichweh, Rudolf. (2013). Regionale Diversifikation und funktionale Differenzierung der Weltgesellschaft. Disponible en: <https://www.fiw.uni-bonn.de/demokratieforschung/personen/stichweh/pdfs/95_stw_regionale-diversifikation-und-funktionale-differenzierung-der-weltgesellschaft.pdf $\geq$.

Stichweh, Rudolf. (2000). Die Weltgesellschaft. Frankfurt: Suhrkamp.

Tau, Victor. (2015). Provincial and local law of the Indies. A research program. In: Duve, Thomas \& Pihlajamaki, Heikki (eds). New horizons in Spanish colonial law. Frankfurt: Max Planck Institute for European Legal History.

Therborn, Göran. (1996). European modernity and beyond. The trajectory of European societies 1945-2000. London: Sage. Trevor-Ropper, Hugh. (I999). The crisis of the seventeenth century. Indianapolis: Liberty Fund.

Valenzuela, Eduardo \& Cousiño, Carlos. (2015). Politización y monetarización en América Latina. Santiago: IES.

Véliz, Claudio. (I970). Obstacles to change in Latin America. London: Oxford University Press.

Weber, David. (2004). Spanish Bourbons and wild Indians. Baylor: Baylor University Press. 


\section{DIRETRIZES PARA UMA SOCIOLOGIA EVOLUTIVA DE DIFERENCIAÇÃO FUNCIONAL NA AMÉRICA LATINA}

Resumo

Nesse artigo, argumentamos que a diferenciação funcional, o modo predominante de organização social da sociedade mundial, não tem sido uma imposição europeia na América, porque, no momento em que as duas sociedades regionais entram em contato, isso ainda não tem lugar. A diferenciação funcional é formada na relação entre Europa e América. Além disso, originam-se redes de múltiplas escalas que coevoluem com diferenciação funcional e dão um caráter particular à diferenciação americana. Com base no conceito de diferenciação funcional de Niklas Luhmann e em uma análise histórico-evolucionária do processo de diferenciação na América, identificamos momentos particulares desse desenvolvimento e suas características centrais. Concluímos que a sociologia latino-americana requer uma abordagem evolutiva da sociedade para descentralizar sua visão do presente, escapar do nacionalismo metodológico que a caracteriza e se transformar na sociologia da sociedade mundial.

\section{GUIDELINES FOR AN EVOLUTIONARY SOCIOLOGY OF FUNCTIONAL DIFFERENTIATION IN LATIN AMERICA}

In this article we argue that functional differentiation, the prevalent form of social organisation in world society, was not a European imposition on America, since it had yet to take place at the moment when the two regional societies came into contact. Functional differentiation emerged from the relationship between Europe and America. Moreover, multilayered networks also became formed, co-evolving with functional differentiation in America and giving a particular character to American differentiation. Setting out from Niklas Luhmann's concept of functional differentiation and a historical-evolutionary analysis of the process of differentiation in America, we identify particular moments of this development and its main features. We conclude that Latin American sociology requires the adoption of an evolutionary approach to society to decentralize its vision from the present, escape the methodological nationalism that characterizes it, and transform itself into the sociology of world society.
Palavras-chave

Diferenciação funcional;

América Latina;

redes;

estratificação;

centro/periferia;

teoria dos sistemas;

Niklas Luhmann.

\section{Keywords}

Functional differentiation; Latin America; networks; stratification; centre/periphery; systems theory; Niklas Luhmann. 